\title{
Proximal Descending Thoracic Aortic Pseudoaneurysm Secondary to Pott's Spine
}

\author{
Irappa Madabhavi, MBBS, MD, DM ${ }^{1}$ Malay Sarkar, MBBS, MD² Chidanand Chauhan, MBBS, MD 3 \\ Mitul Modi, MBBS, DCP, MD 4
}
1 Department of Medical and Pediatric Oncology and Hematology, Kerudi Cancer Hospital, Bagalkot, Karnataka, India
2 Department of Pulmonary Medicine, Indira Gandhi Medical College, Shimla, Himachal Pradesh, India
${ }^{3}$ Department of Radiology, Indira Gandhi Medical College, Shimla, Himachal Pradesh, India
${ }^{4}$ Department of Pathology, Gujarat Cancer and Research Institute, Ahmedabad, Gujarat, India

\begin{abstract}
Address for correspondence Irappa Madabhavi, MBBS, MD, DM, Department of Medical and Pediatric Oncology and Hematology, Kerudi Cancer Hospital, Bagalkot-587101, Karnataka, India (e-mail: irappamadabhavi@gmail.com).
\end{abstract}

AORTA 2020;8:35-37.
Abstract
Keywords
- tuberculosis
- vertebra
- aneurysm
- antitubercular treatment

Tuberculous pseudoaneurysm of the descending thoracic aorta is quite rare, lifethreatening, and fatal if not diagnosed in time. This lesion exposes patients to a very high risk of unpredictable rupture. We describe a case of tuberculous pseudoaneurysm of the aorta in association with tuberculosis of the spine (Pott's spine). A 73-year-old man presented with a 2-month history of back pain. Chest roentgenography and contrast-enhanced computed tomography showed a descending thoracic aortic pseudoaneurysm with destruction of the fourth and fifth thoracic vertebrae (T4-T5). We suspected that the pseudoaneurysm was due to direct extension of tuberculous vertebral osteomyelitis. The patient was managed with antituberculous chemotherapy. The post-antitubercular therapy course was uneventful and he remained well 12 months after completion of treatment.

\section{Introduction}

Tubercular pseudoaneurysm of the descending thoracic aorta is a rare and potentially fatal complication of tuberculosis (TB). However, not all cases of aortic involvement by TB lead to aneurysm formation. ${ }^{1}$

\section{Case Presentation}

A 73-year-old man presented to hospital with a 2-month history of dull back pain. He had no prior history of pulmonary TB. He was not a smoker and had no history of angina, hypertension, dyslipidemia, or ischemic stroke. On admission to hospital, physical examination and laboratory values were normal limits for his age. No causative pathogens were cultured from blood or sputum. Chest roentgenography and magnetic

received

June 13, 2018

accepted after revision

November 18, 2019
DOI https://doi.org/

10.1055/s-0040-1701212. ISSN 2325-4637. resonance imaging showed a descending thoracic aortic aneurysm with severe destruction of the fourth and fifth thoracic vertebral bodies with pre- and paravertebral soft tissue components ( - Fig. 1). Contrast-enhanced computed tomographic angiography (CTA) and three-dimensional images reconstruction confirmed a saccular pseudoaneurysm measuring $52 \times 45 \times 35 \mathrm{~mm}$ involving the posterior wall of the proximal descending thoracic aorta with severe destruction of the fourth and fifth thoracic vertebral bodies ( - Fig. 2A,B).

TB aneurysm was confirmed with Mantoux test of more than $15-\mathrm{mm}$ induration, raised erythrocyte sedimentation rate of $80 \mathrm{~mm} / \mathrm{h}$, and polymerase chain reaction for Mycobacterium tuberculosis deoxyribonucleic acid (DNA) was positive. Since most thoracic aortic aneurysms with corresponding vertebral erosion are associated with mycotic infection, especially $\mathrm{TB}$, we suspected this was a tuberculous aortic aneurysm
Copyright $\odot 2020$ by Thieme Medical Publishers, Inc., 333 Seventh Avenue, New York, NY 10001, USA. Tel: +1(212) 584-4662.
License terms

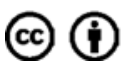




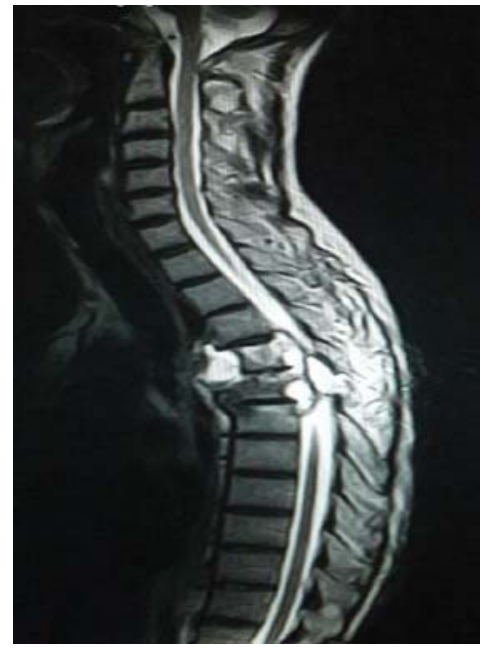

Fig. 1 Magnetic resonance imaging showing abnormal signal changes in the T4 and T5 disks and adjacent vertebral bodies with associated prevertebral and epidural soft tissue abnormalities.

and initiated antituberculous chemotherapy with isoniazid $(0.3 \mathrm{~g} / \mathrm{d})$, rifampin $(0.45 \mathrm{~g} / \mathrm{d})$, ethambutol $(0.75 \mathrm{~g} / \mathrm{d})$, and pyrazinamide $(0.5 \mathrm{~g} / \mathrm{d})$ for 9 months.

In view of the old age and poor performance status, after discussion regarding the cardiovascular surgical interventional options, including thoracotomy and cardiopulmonary bypass and also endovascular grafting for this extensive pseudoaneurysm, the patient and the family opted for sole medical management via antitubercular treatment.

We experienced an exceedingly rare case of descending thoracic aortic pseudoaneurysm with severe vertebral erosion at T4 and T5 level, which was likely caused by direct extension of tuberculous vertebral osteomyelitis. Treatment with combined antituberculous chemotherapy was successful, and the patient remains well 12 months after the completion of treatment. After 2-years follow-up, his symptoms and signs disappeared without pseudoaneurysm recurrence by the computed tomography examination.

\section{Discussion}

Tubercular pseudoaneurysm of the descending thoracic aorta is a rare and a potentially fatal complication of TB. However, not all cases of aortic involvement by TB lead to aneurysm formation. ${ }^{1}$ There are four mechanisms by which tubercle bacilli may reach the aortic wall: (1) Direct implantation of the bacilli on the internal surface of the aortic wall may occur. Normally, the intima wall is resistant to infection. However, an atherosclerotic patch may increase the risk of direct implantation. Schmorl ${ }^{2}$ detected of tuberculosis bacilli on atheromatous ulcers in autopsies in 5 of 123 patients with acute miliary TB; (2) The bacilli may be carried to the adventitia or media by the vasa vasorum; (3) Involvement of the vessel wall may occur indirectly via the lymphatics ${ }^{3}$; (4) Direct extension from a neighboring tuberculous lymph node, abscess, or bone may occur. This has been reported in the literature as the most common cause (75\%). A contiguous focus of lymph nodes has been described in $63 \%$ of the cases, whereas, paraspinal abscess, lung, pericardium, vertebrae, and prostate are the source of infection in $37 \%$ cases.

TB mainly involves the thoracic or the abdominal aorta. ${ }^{4}$ Involvement of the descending thoracic aorta in association with vertebral TB is rare. Clinically, TB of the aorta remains silent until some major complications develop. The following clinical features of tuberculous aortic aneurysm have been described in the literature. These include persistent chest, abdominal, or back pain, hypovolemic shock or other evidence of major bleeding, particularly into the lung or gastrointestinal
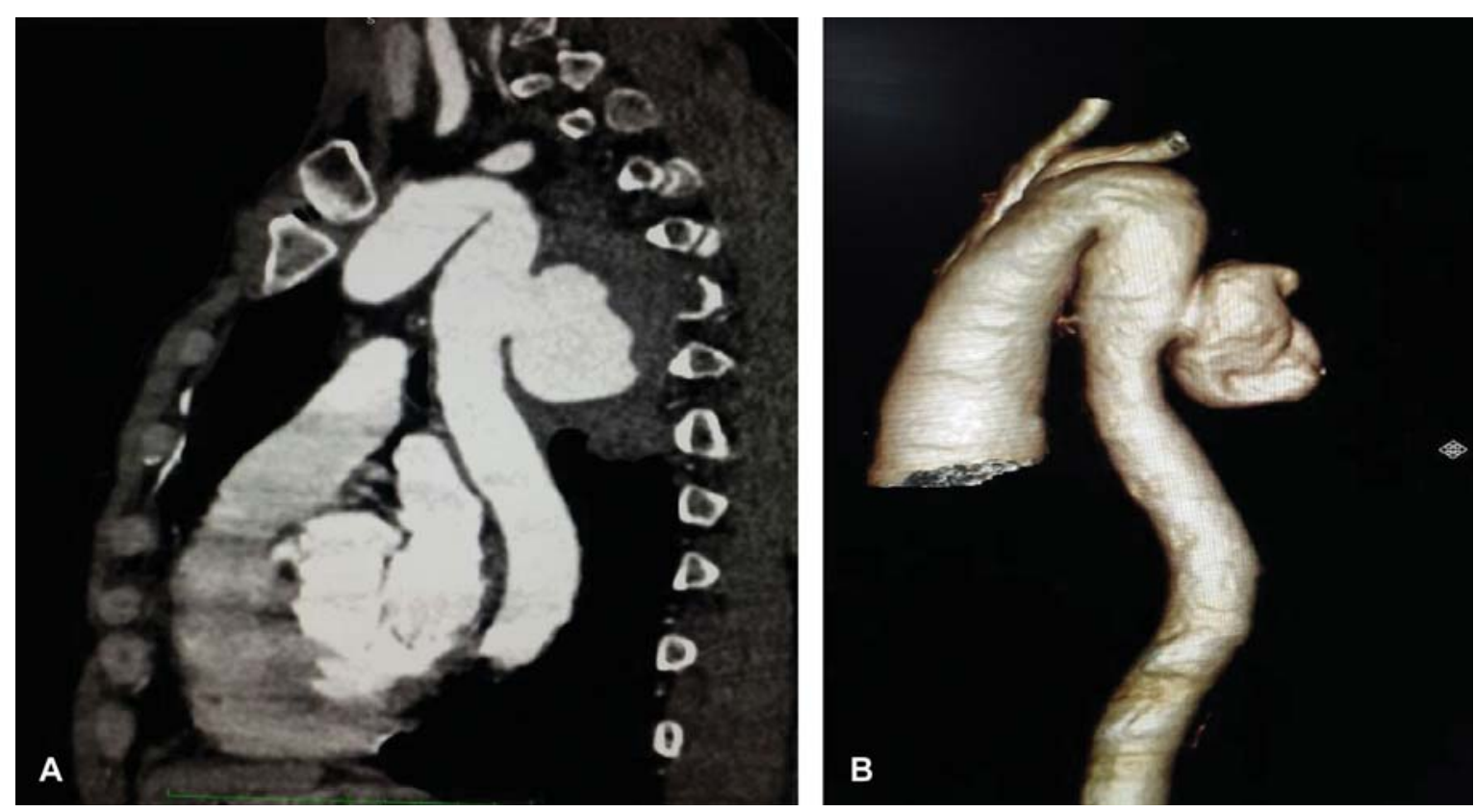

Fig. 2 (A) Sagittal and (B) three-dimensional reconstruction of contrast-enhanced reveals an aortic pseudoaneurysm measuring $52 \times 45 \times 35 \mathrm{~mm}$ involving the posterior wall of the proximal descending thoracic aorta. 
tract, but also into the pleural space, peritoneal cavity, retroperitoneum, or pericardial space, and a palpable or radiographically visible para-aortic mass, especially if expanding or pulsatile. ${ }^{5}$ Contrast-enhanced computed tomography and magnetic resonance angiography are the modalities commonly used in the detection of tuberculous aortic aneurysm. The characteristic features of tuberculous aortic aneurysm are the absence of pulsation ${ }^{6}$ and contrast filling. ${ }^{7}$ Other features supporting TB are the presence of a false aneurysm, noninvolvement of the ascending aorta, and radiological presence of a contiguous focus of disease on computed tomography scan. ${ }^{6,8}$

Currently, surgery combined with simultaneous anti-TB drug treatment should be used for the disease, especially while considering that no evidence exists to show only antiTB drug treatment or surgery alone can achieve a cure. Common surgical methods include the following: vascular lesion removal and synthetic vascular replacement; extraanatomical reconstruction; direct suture closure or patch repair; and endovascular stent-graft exclusion or endovascular aneurysm repair. Currently, the most common surgical approach involves the resection of the diseased segment, removal of the surrounding necrotic tissues, and reconstruction of the distal vessel using a graft from the noninfectious region far from the infected areas (anatomical bypass). ${ }^{9,10}$

In summary, we report the case of a 73-year-old male with tuberculous vertebral and pseudoaneurysm formation in the proximal descending thoracic aorta. Pseudoaneurysm is a rare complication of infection by Mycobacterium tuberculosis. Contrast-enhanced CTA is the investigation of choice for proper delineation of the aneurysm. Treatment should include a full course of antituberculous chemotherapy and directed vascular surgery. If unrecognized, these aneurysms may rupture, possibly with lethal consequences.
Funding

None.

Conflict of Interest

The authors declare no conflict of interest related to this article.

Acknowledgment

None.

\section{References}

1 Silbergleit A, Arbulu A, Defever BA, Nedwicki EG. Tuberculous aortitis: surgical resection of ruptured abdominal false aneurysm. JAMA 1965;193:333-335

2 Schmorl G. Zur Frage der Genese der Lungertuberkulose. Munch Med Wochenschr 1902;49:1379-1419

3 Smith G, Hutchison HE. Lymph borne infection and aneurysm formation. Surg Gynecol Obstet 1957;104(06):722-726

4 Choudhary SK, Bhan A, Talwar S, Goyal M, Sharma S, Venugopal P. Tubercular pseudoaneurysms of aorta. Ann Thorac Surg 2001;72 (04):1239-1244

5 Volini FI, Olfield RC Jr, Thompson JR, Kent G, Park O. Tuberculosis of the aorta. JAMA 1962;181:78-83

6 Long R, Guzman R, Greenberg H, Safneck J, Hershfield E. Tuberculous mycotic aneurysm of the aorta: review of published medical and surgical experience. Chest 1999;115(02):522-531

7 Yeoh CB, Ford JM, Garret R. Tuberculous pseudoaneurysm of descending thoracic aorta. Surgical treatment. Arch Surg 1963;86:318-322

8 Ringswald M, Roy TM. Synchronous mycotic aneurysms secondary to tuberculosis. J Ky Med Assoc 1989;87(07):320-324

9 Zhang C, Chen B, Gu Y, et al. Tuberculous abdominal aortic pseudoaneurysm with renal and vertebral tuberculosis: a case and literature review. J Infect Dev Ctries 2014;8(09):1216-1221

10 Xue J, Yao Y, Liu L. Treatment of tuberculous aortic pseudoaneurysm associated with vertebral tuberculosis: a case series and a literature review. Medicine (Baltimore) 2018;97(15):e0382 